\title{
Granulocyte-Macrophage Colony-Stimulating Factor Modulates Rapid Eye Movement (REM) Sleep and Non-REM Sleep in Rats
}

\author{
Mayumi Kimura, ${ }^{1}$ Tohru Kodama, ${ }^{2}$ M. Cecilia Aguila, ${ }^{3}$ Shi-Qing Zhang, ${ }^{1}$ and Shojiro Inoué ${ }^{1}$ \\ ${ }^{1}$ Department of Biocybernetics, Institute of Biomaterials and Bioengineering, Tokyo Medical and Dental University, Tokyo \\ 101-0062, Japan 2Department of Psychology, Tokyo Metropolitan Institute for Neuroscience, Tokyo 183-8526, Japan, and \\ 3 Department of Neurology, University of Miami, Miami, Florida 33125
}

\begin{abstract}
Granulocyte-macrophage colony-stimulating factor (GM-CSF) is a hematopoietic cytokine that may affect various functions of the CNS because the molecule and its receptors are expressed in the brain. The present study examines the effects of GM-CSF on sleep using rats and the secretion of three neurotransmitters/ hormones that are involved in sleep regulation. When infused intracerebroventricularly at doses as low as 10 pmol for $10 \mathrm{hr}$ during the dark period, GM-CSF promoted predominantly rapid eye movement (REM) sleep and moderate amounts of non-REM sleep without eliciting fever. An injection of GM-CSF (3.0 pmol) into the arcuate nucleus increased the release of nitric oxide (NO) from the hypothalamus but did not alter plasma levels of growth
\end{abstract}

hormone. The release of somatostatin (SRIF) from the medial basal hypothalamus was stimulated by $1 \times 10^{-11} \mathrm{M}$ GM-CSF. These findings indicated that centrally administered GM-CSF stimulates SRIF release through activation of the NO system in the hypothalamus. Because SRIF promotes REM sleep, it may also mediate the effects of GM-CSF on REM sleep. The present study indicates a novel central effect of GM-CSF that modulates sleep, supporting the notion that hematopoietic cytokines also play roles in the CNS.

Key words: sleep; cytokine; hypothalamus; nitric oxide; somatostatin; rat
Cytokines are products of activated immune and mesenchymal cells, and their roles in infection, inflammation, and acute-phase responses have been extensively studied. However, cytokines are multifunctional polypeptides rather than simple immune factors. Studies of interleukin 1 (IL-1) and tumor necrosis factor- $\alpha$ (TNF- $\alpha$ ) have revealed that cytokines interfere with various functions of the CNS (Rothwell and Hopkins, 1995). Fever and hypersomnolence, which are caused by proinflammatory cytokines produced by host immune cells affecting the brain, often accompany infectious diseases (Krueger and Majde, 1994).

Granulocyte-macrophage colony-stimulating factor (GM-CSF) is a $14-35 \mathrm{kDa}$ glycoprotein that was originally described as a hematopoietic cytokine because it potently stimulates myeloid progenitor cells in vitro to proliferate and differentiate into mature macrophages and polymorphonuclear leukocytes (Burgess and Metcalf, 1980). However, serum levels of GM-CSF can be elevated in response to infectious stimuli (Kuhns et al., 1995). Significant amounts of GM-CSF are synthesized in the uterus at the beginning of pregnancy (Robertson and Seamark, 1992). Furthermore GMCSF affects the release of adenocorticotropic hormone (Komorowski et al., 1996) and luteinizing hormone (LH) (Kimura et al., 1996a) from the anterior pituitary gland. Therefore GM-CSF probably plays a role in the immune system as well as in endocrine regulation, like IL-1.

Increasing evidence suggests that the CNS is also a target of GM-CSF, because GM-CSF mRNA is synthesized in astrocytes (Malipiero et al., 1990; Aloisi et al., 1992) and GM-CSF receptors are located in microglia (Fischer et al., 1993; Sawada et al., 1993) and oligodendrocytes (Baldwin et al., 1993). The release of lutein-

Received Feb. 22, 2000; revised April 20, 2000; accepted May 4, 2000.

This work was supported in part by a grant-in-aid from the Ministry of Education, Science, Sports, and Culture, Japan, to M.K. (08771311 and 11671600) and a Special Coordination Fund for Promoting Science and Technology from the Science and Technology Agency, Prime Minister's Office to S.I. We thank Ms. Yoshiko Honda for her excellent assistance and Dr. Ferenc Obál Jr. for valuable comments regarding this manuscript.

Correspondence should be addressed to Dr. Mayumi Kimura, Department of Biocybernetics, Institute of Biomaterials and Bioengineering, Tokyo Medical and Dental University, 2-3-10 Kanda-Surugadai, Chiyoda-ku, Tokyo 101-0062, Japan. E-mail: mkimura@i-mde.tmd.ac.jp.

Copyright (C) 2000 Society for Neuroscience $0270-6474 / 00 / 205544-08 \$ 15.00 / 0$ izing hormone-releasing hormone (LHRH) from the hypothalamus is suppressed by GM-CSF (Kimura et al., 1997). Gliosis in the brain is induced by GM-CSF released from brain tumor cells (Giulian et al., 1994), and GM-CSF is required for neural development in neonates (Mehler and Kessler, 1997). The system that transports GM-CSF from the blood to the brain is saturable (McLay et al., 1997). Clinical trials have shown occasional febrile responses associated with GM-CSF therapy (Lieschke et al., 1990; Bokemeyer et al., 1993). However, neither the behavioral nor somnogenic effects of hematopoietic cytokines have been thoroughly studied, although clinicians should understand the potential for unpredictable central side effects generated by these cytokines.

The present study examines the effects of GM-CSF on sleep using an experimental animal model. Other cytokines affect the hypothalamic-pituitary axes (McCann et al., 1994) and many hypothalamic and pituitary hormones are implicated in sleep regulation (Steiger et al., 1998). Therefore, we investigated whether or not GM-CSF modulates sleep through interaction with the neuroendocrine system. The results showed that GM-CSF exerts a somnogenic action, indicating that hematopoietic cytokines are involved in CNS function.

\section{MATERIALS AND METHODS}

Chemicals. Recombinant murine GM-CSF (14.5 kDa; carrier-free) and antibody against murine GM-CSF (anti-GM-CSF) were purchased from R \& D Systems (Minneapolis, MN). The control experiment included GMCSF inactivated by immersion in boiling water for $5 \mathrm{~min}$. Reagents required for nitrite/nitrate (NOx) analysis were obtained from Eicom (Kyoto, Japan). Plasma levels of growth hormone $(\mathrm{GH})$ were measured by a rat GH enzyme immunoassay (EIA) (Amersham International Plc, Buckinghamshire, UK). Standard rat somatostatin (SRIF) for radioimmunoassay (RIA) was purchased from Peninsula Laboratories (Belmont, CA). Other chemicals were purchased from Sigma (St. Louis, MO).

Animals. Adult male Sprague Dawley rats, weighing 280-380 gm, were kept in groups on a $12 \mathrm{hr}$ light/dark cycle (lights on at 6:00 or 8:00 A.M.; off at 6:00 or 8:00 P.M.) in a constant environment at $25 \pm 1{ }^{\circ} \mathrm{C}$ and $60 \pm$ $6 \%$ relative humidity. Rat chow and tap water were accessible ad libitum. All animal experiments in the present study were approved by the Animal Care and Use Committee at Tokyo Medical and Dental University.

Surgery. The rats were anesthetized with an intraperitoneal injection of sodium pentobarbital $(50 \mathrm{mg} / \mathrm{kg})$, then fixed on a stereotaxic apparatus. Three electroencephalographic (EEG) and two electromyographic (EMG) electrodes and a thermistor were implanted as described previously (Inoué et al., 1985). Briefly, the EEG electrodes were gold-plated stainless steel 
screws placed through the skull on the frontal and occipital cortex. The EMG electrodes constructed from hypodermic stainless steel needles were inserted into the cervical portion of the trapezius muscle. The thermistor electrode (G-1E model; Toho Electric Company, Tokyo, Japan) to record brain temperature ( $\mathrm{T} b r$ ) was placed in the thalamus at a depth of $4 \mathrm{~mm}$ from the skull. To achieve continuous central infusion, an intracerebroventricular cannula $[0.35 \mathrm{~mm}$ inner diameter (i.d.)] was inserted into the third ventricle $(3 \mathrm{~V})$. In another group of rats, an intracerebral guide cannula (type MI-AG; $0.57 \mathrm{~mm}$ i.d.) for a microdialysis probe (MI-AI12-01; Eicom) and local injection was inserted into the arcuate nucleus (Arc) of the hypothalamus, according to the brain atlas of Paxinos and Watson (1997). The coordinates of the guide tip were $3.5 \mathrm{~mm}$ posterior and $9.0 \mathrm{~mm}$ ventral to the bregma and $0.2 \mathrm{~mm}$ lateral to the midline. All electrodes and cannulae were permanently affixed to the skull using dental acrylic resin. During and at the end of surgery, a total of 40,000 $\mathrm{U}$ of penicillin G potassium (Meiji Pharmaceutical Company, Tokyo, Japan) was subcutaneously and locally applied to the incision. After surgery, the rats were individually caged in the same environment as before. Experiments were started $\sim 1$ week after surgery.

Sleep recordings and intracerebroventricular infusion. After recovery, the rats implanted with intracerebroventricular cannulae were placed into individual sleep-recording cages in a sound-attenuated, electromagnetically shielded chamber. The lead wires of the EEG and EMG electrodes and of the brain thermistor were connected to a multichannel amplifier (MEG-6116; Nihon, Kohden, Tokyo, Japan) or to a thermistor amplifier (ELMEC, Tokyo, Japan) and a high-speed analog-to-digital converter (EC-2390B, ELMEC), via a feed-through slip ring (CAY-675; Airflyte Electronics Company, Bayonne, NJ) fixed above the cage. Signals of EEG, EMG, and $\mathrm{T} b r$ were digitized and graphically displayed by a PC-9821 Ap2/U2 (NEC, Tokyo, Japan) equipped with an automatic sleep-wake analyzer (ELMEC). Polygraphic data were stored on a computer every 30 min. Vigilance states were automatically classified over periods of $12 \mathrm{sec}$ as non-rapid eye movement (non-REM) sleep, REM sleep, or wakefulness as described elsewhere (Inoué et al., 1985). In short, non-REM sleep was characterized by high-amplitude low-frequency EEG waves and an intermediate level of EMG activity with a gradual decrease in Tbr. REM sleep was identified by low-amplitude, high-frequency EEG waves and a lack of EMG activity, except for phasic muscle twitches with a rapid increase in Tbr. Wakefulness was characterized by low-amplitude, high-frequency EEG waves and high, variable amplitude EMG activity with a gradual increase in Tbr. The automated classification was visually confirmed and restored in edited forms.

After recovery from surgery, a continuous intracerebroventricular infusion of saline was initiated $(10 \mu \mathrm{l} / \mathrm{hr})$ before recording. The intracerebroventricular cannula was connected to extended polyethylene tubing (PE10) attached to an infusion pump through the slip ring with Teflon connecting tubing $(0.5 \mathrm{~mm}$ i.d. $)$. Thus, unrestrained movement of the rats was guaranteed during the study. After the rats were acclimatized to the intracerebroventricular infusion, a standardized $3 \mathrm{~d}$ assay was implemented. Day 1 was assigned as the control day with an infusion of saline only. Day 2 was assigned as the test day when the rats received either GM-CSF (1.0 and $10 \mathrm{pmol}), 10 \mathrm{pmol}$ of heat-inactivated GM-CSF, or anti-GM-CSF $(10 \mu \mathrm{g})$ dissolved in $100 \mu \mathrm{l}$ of saline for $10 \mathrm{hr}$ from the onset of the dark period (at 8:00 P.M.). Day 3 was assigned as the recovery day with a continuous infusion of saline. Polygraphic recordings of EEG, EMG, and Tbr were obtained over the entire $3 \mathrm{~d}$ from the beginning of the light period on day 1 . If the pattern of sleep-wake cycles was irregular during the baseline recordings on day 1 , the animal was discarded.

Microinjection of GM-CSF into the Arc and nitric oxide measurement. During recovery from the surgery, the rats implanted with the guide cannula were moved to individual cages, where locomotor activity was continuously monitored using an infrared detecting device (NS-AS01; Neuroscience, Tokyo, Japan) directly connected to a computer for automated data processing. When locomotion returned to the basic nocturnal pattern, local injection was initiated. Under halothane anesthesia, a microinjection cannula $(0.15 \mathrm{~mm}$ i.d. $)$ attached to the probe for microdialysis was placed into the guide cannula at least $3 \mathrm{hr}$ before injection. Lead tubing from the probe outlet and the microinjection cannula connected to the autosampler of the HPLC system and a $1.0 \mu$ l Hamilton syringe, respectively, were extended over the cage so as not to restrain free movement during the experimental period. At a dose of 3 pmol dissolved in $0.5 \mu \mathrm{l}$ of saline, GM-CSF was injected for $1 \mathrm{~min}$ into the Arc, and dialysates from the Arc were collected every $10 \mathrm{~min}$ for $1 \mathrm{hr}$ before and $6 \mathrm{hr}$ after injection. NOx in the dialysates was separated by liquid column $(4.6 \times 50 \mathrm{~mm}$ NO-PAK, Eicom) chromatography into $\mathrm{NO}_{2}$ and $\mathrm{NO}_{3}$, then measured individually using the Griess method (detection limit, 0.1 pmol) (Yamada et al., 1997). The same rats were randomly injected with saline before or after receiving GM-CSF to provide control values, and baseline changes in NOx in the dialysates were also measured. To provide a positive control, norepinephrine (NE) was injected to stimulate the release of nitric oxide (NO) from the Arc (Agullo and Garcia, 1991). Only those rats that responded to NE were studied. All injections were administered at $\sim 12: 00$ P.M. (lights on at 6:00 A.M.).

Microinjection of GM-CSF and analyses of plasma GH. Rats implanted with guide cannulae that were not used in the above experiment, were anesthetized with pentobarbital sodium after recovery from the first operation. For continuous blood collection, an indwelling catheter was placed into the right atrium of the heart through the right external jugular vein. Experiments were performed on the following day. Either GM-CSF (3.0 pmol in $0.5 \mu$ l of saline) or saline $(0.5 \mu \mathrm{l})$ as a control vehicle was injected into the Arc $1 \mathrm{hr}$ after connecting the extension tubing (PE50) to the jugular catheter. The microinjection cannula was placed in the guide cannula as described above. Blood $(0.3 \mathrm{ml})$ was withdrawn through the catheter immediately before (time 0 ) and every $10 \mathrm{~min}$ after the local injection for $2 \mathrm{hr}$ without disturbing the animals. After each blood sampling, an equal volume of heparin solution $(50 \mathrm{U} / \mathrm{ml}$ saline $)$ was injected to maintain blood volume. Blood samples were immediately separated into plasma and red blood cells by centrifugation for $15 \mathrm{~min}$ at $4500 \times \mathrm{g}$ at $4^{\circ} \mathrm{C}$. Plasma samples were stored at $-20^{\circ} \mathrm{C}$ until assay.

Somatostatin release in vitro. Immediately after decapitation, the medial basal hypothalamus (MBH) was dissected from the brain as described previously (Karanth et al., 1993). The MBH explants 2-mm-long rostrocaudally, $2 \mathrm{~mm}$ in depth, extended $0.5 \mathrm{~mm}$ bilaterally from the midline and encompassed the median eminence, pituitary stalk, Arc, and periventricular and ventromedial nuclei. Each explant was incubated for $1 \mathrm{hr}$ in $0.5 \mathrm{ml}$ of Krebs'-Ringer's solution of bicarbonate (KRB) glucose (100 mg\%) buffer, $\mathrm{pH} 7.4$, containing $0.1 \%$ of bovine serum albumin in a Dubnoff metabolic shaker (60 cycles/min) in an atmosphere of $95 \% \mathrm{O}_{2}$ and $5 \%$ $\mathrm{CO}_{2}$. Thereafter, the medium was discarded, and the tissues were incubated for $0.5 \mathrm{hr}$ either in fresh medium (control) or medium containing $1 \times$ $10^{-12}-1 \times 10^{-8} \mathrm{M}$ GM-CSF. Media were then boiled for $10 \mathrm{~min}$ in a water bath to prevent enzymatic degradation and stored at $-20^{\circ} \mathrm{C}$. The quantity of SRIF present in the incubation media was determined by RIA as described elsewhere (Arimura et al., 1975; Aguila and McCann, 1985. Aguila et al., 1991), with minor modifications. The tracer was $\left[{ }^{125} \mathrm{I}\right] \mathrm{Tyr}^{1}$ SRIF and synthetic SRIF was the reference standard. The highly specific antiserum (RC-IIC) provided by Dr. L. De Palatis (Neoprobe Company, Dublin, $\mathrm{OH}$ ) was initially diluted 1:50,000. In brief, the antiserum crossreacts $100 \%$ with synthetic SRIF (SRIF-14) and $40 \%$ with SRIF-28; 50 pg of SRIF displaced $50 \%$ of the maximal binding. It does not cross-react with LHRH, vasoactive intestinal peptide, thyrotropin-releasing hormone, $\alpha$ melanocyte-stimulating hormone, cholecystokinin- 8 , substance $\mathrm{P}$, and $\beta$-endorphin. The sensitivity of the SRIF assay is $3.1 \mathrm{pg} / \mathrm{tube}$, and the intra-assay and inter-assay coefficients of variation were $\sim 5.6$ and $12.5 \%$.

Data analyses. Time spent in non-REM sleep and REM sleep was calculated in 1,2 , or $12 \mathrm{hr}$ averages corresponding to the light/dark period for each rat. The Tbr values (collected at $3 \mathrm{~min}$ intervals) were averaged for $1 \mathrm{hr}$. The effects of GM-CSF on sleep, Tbr, NOx, and plasma GH were compared with those of saline on the control day and statistically analyzed using parametric two-way ANOVA, or two-way ANOVA for repeated measures. If the $F$ value reached statistical significance, the StudentNewman-Keuls multiple comparison test was further applied for post hoc analysis. The effects of GM-CSF on SRIF release in vitro were compared with of the KRB control by one-way ANOVA and post hoc Dunnett's test. A level of $p<0.05$ was considered significant.

\section{RESULTS}

\section{Effects of intracerebroventricularly infused GM-CSF on sleep and Tbr}

Circadian variation in sleep-wake patterns and Tbr were altered in a dose-dependent manner by GM-CSF (Table 1). At a dose of 10 pmol, GM-CSF significantly increased both nocturnal non-REM sleep and REM sleep on day 2 (Table 1, Fig. 1, left). Increased non-REM sleep was evident for $3 \mathrm{hr}$ at the beginning of the inf usion period $(63.7 \%$ above baseline; $p<0.05)$, whereas REM sleep was enhanced for $10 \mathrm{hr}$ during the entire infusion period (158.3\% above baseline; $p<0.05)$. Even after the GM-CSF infusion was terminated, REM sleep remained significantly elevated for $3 \mathrm{hr}$ in the light $(103.8 \%$ above baseline; $p<0.05)$ and $3 \mathrm{hr}$ in the dark period (145.7\% above baseline; $p<0.05$ ) on day 3 (Fig. 1, right). Enhanced non-REM and REM sleep in response to GMCSF were caused by elevated numbers of each episode rather than prolonged episode duration (Table 2). The Tbr levels slightly increased during the dark period on day 2 (Table 1). Febrile responses that occurred at the end of the infusion period were not associated with sleep responses (Fig. 1, left). On day 3, although Tbr was slightly above the baseline, these changes in Tbr were not statistically significant (Fig. 1, right). Neither the low dose $(1.0 \mathrm{pmol})$ nor heat-inactivated GM-CSF affected sleep or Tbr (Table 1).

Anti-GM-CSF $(10 \mu \mathrm{g})$ did not significantly affect sleep on the day of infusion (day 2) (Fig. 2, left). On day 3, however, nocturnal sleep patterns were altered. Non-REM sleep and REM sleep (14.7 and $44.1 \%$ less than baseline; $p<0.05$, respectively) were significantly decreased in the middle of the dark period between 11:00 P.M. and 4:00 A.M. (Fig. 2, right). These changes were attributable to a decreased number of episodes rather than the decreased 
Table 1. Effects of GM-CSF on nocturnal sleep and brain temperature relative to the baseline values on day 1

\begin{tabular}{|c|c|c|c|c|c|c|}
\hline \multirow[b]{2}{*}{ GM-CSF } & \multicolumn{2}{|c|}{ Non-REM Sleep $(\%)^{a}$} & \multicolumn{2}{|c|}{ REM Sleep $(\%)^{a}$} & \multicolumn{2}{|l|}{$\operatorname{Tbr}\left( \pm^{\circ} \mathrm{C}\right)^{b}$} \\
\hline & Day 2 & Day 3 & Day 2 & Day 3 & Day 2 & Day 3 \\
\hline $1 \mathrm{pmol}(n=7)$ & $106.9 \pm 6.2$ & $99.2 \pm 3.5$ & $106.8 \pm 11.0$ & $117.6 \pm 13.7$ & $+0.00 \pm 0.06$ & $-0.07 \pm 0.04$ \\
\hline $10 \mathrm{pmol}(n=7)$ & $126.6 \pm 10.1^{*}$ & $103.1 \pm 6.4$ & $303.2 \pm 61.7^{*}$ & $243.6 \pm 49.0^{*}$ & $+0.19 \pm 0.04^{*}$ & $+0.22 \pm 0.18$ \\
\hline Heated $(n=6)$ & $96.2 \pm 2.2$ & $95.7 \pm 4.4$ & $94.8 \pm 8.2$ & $92.8 \pm 7.2$ & $+0.09 \pm 0.03$ & $+0.00 \pm 0.06$ \\
\hline Antibody $(n=5)$ & $104.7 \pm 5.2$ & $83.0 \pm 8.0^{*}$ & $91.1 \pm 5.6$ & $63.1 \pm 7.6^{*}$ & $+0.09 \pm 0.01$ & $+0.09 \pm 0.05$ \\
\hline
\end{tabular}

Values are means \pm SE.

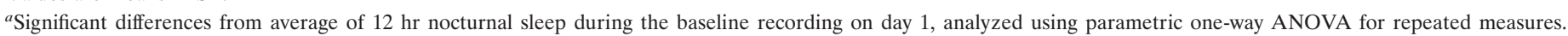
*Statistical significance according to Dunnett's procedure as post hoc analysis $(p<0.05)$.

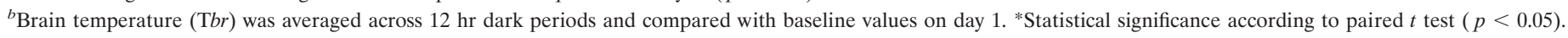

Figure 1. Effects of GM-CSF (10 pmol) on sleep and brain temperature (Tbr). Data points indicate $1 \mathrm{hr}$ averages of changes in $\operatorname{Tbr}(n=6)$ and time spent in non-REM sleep (non-REMS) and REM sleep (REMS) $(n=7)$. Each symbol represents mean $\pm \mathrm{SE}$ (white, day 1; black, day 2; gray, day 3). Horizontal gray bar indicates GM-CSF inf usion for $10 \mathrm{hr}$ on day 2; otherwise the rats were infused intracerebroventricularly with saline during the entire $3 \mathrm{~d}$ period. Horizontal open bar, Light period; horizontal hatched $b a r$, dark period. ANOVA for Tbr between days 1 and 2 during the dark period, $F_{(1,5)}=16.15$; $p<0.01$; ANOVA for non-REMS between days 1 and 2 during postinfusion $\mathrm{hr} 1-3, F_{(1,6)}=$ $26.57 ; p<0.01$; for REMS between day 1 versus days 2 and 3 during the dark period, $F_{(2,12)}=$ $13.58 ; p<0.001$; for REMS between days 1 and 2 versus day 3 during the light period, $F_{(2,12)}=$ $8.12 ; p<0.01$.
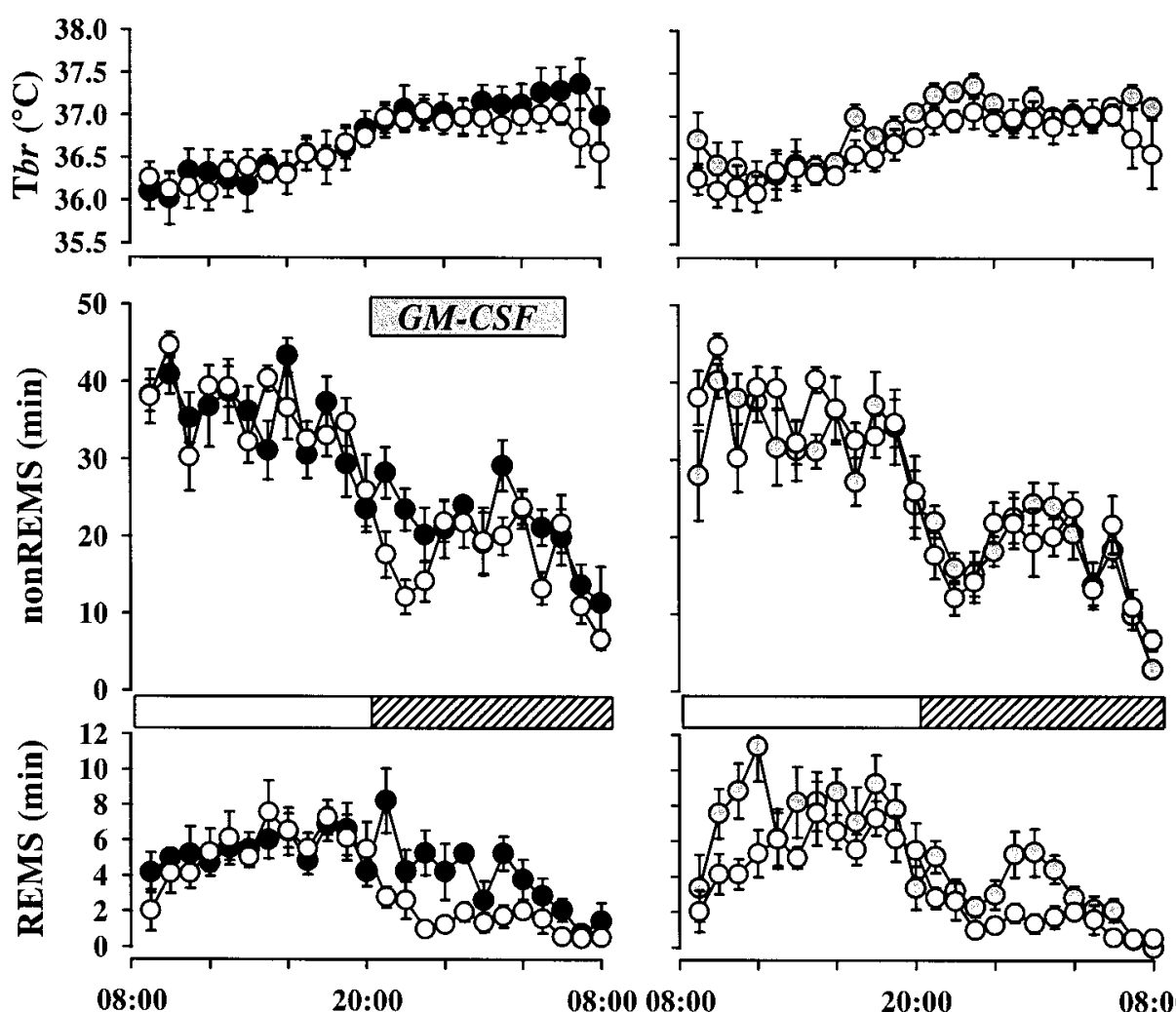

08:00

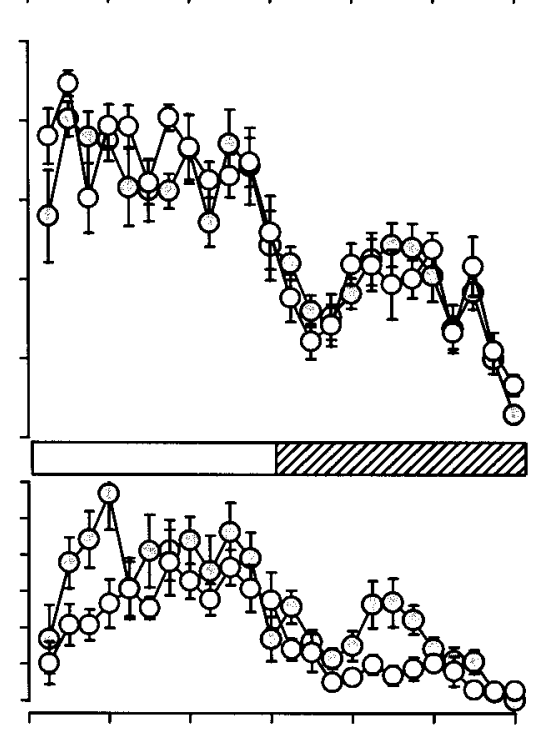

Time of Day (h)

20:00

08:00

Time of Day (h) episode duration (Table 2). Significant changes in Tbr were not induced by anti-GM-CSF on either of days 2 or 3 .

\section{Effects of locally injected GM-CSF on NO release from the Arc}

Immediately after NE (20 mM) was injected locally into the hypothalamus, NO release from the Arc was significantly stimulated (Fig. 3, top). The levels of increased NOx in the Arc returned to the baseline within 30-40 min. No changes in behavior or locomotor activity were visible in response to the hypothalamic injection of NE.

GM-CSF at 3.0 pmol also stimulated NO release from the Arc in the hypothalamus (Fig. 3). Compared with basal release after control saline injection, levels of NOx in the Arc started to elevate $50 \mathrm{~min}$ after GM-CSF injection, and significant increases in the release of NOx peaked by $3 \mathrm{hr}$ after injection (40\% above preinjection baseline; $p<0.05)$. The levels of NOx remained increased during the entire $6 \mathrm{hr}$ sampling period. With respect to behavioral changes, GM-CSF injected into the Arc reduced the numbers of locomotor activity during the dark period, compared with the nocturnal patterns as shown on the day of saline injection (Fig. 4). Suppression of locomotor activity in response to the hypothalamic injection of GM-CSF returned to normal by the onset of the next light period.

\section{Effects of locally injected GM-CSF on plasma levels of $\mathbf{G H}$}

Similarly to the experiments described above, GM-CSF (3.0 pmol) was injected into the Arc of the hypothalamus to determine whether or not locally injected GM-CSF affects plasma levels of $\mathrm{GH}$. After injecting saline into the Arc, pulsatile release of $\mathrm{GH}$ was normal. There were four peaks of elevated $\mathrm{GH}$ during the $2 \mathrm{hr}$ sampling period. The hypothalamic injection of GM-CSF did not affect plasma levels of GH (Fig. 5). After the GM-CSF injection, $\mathrm{GH}$ was released in a pulsatile manner in a manner similar to that after the control injection. The basal release of GH and the number of peaks did not significantly differ from those after the saline injection, although the magnitude of the pulsatile release tended to be a little lower than the control level.

\section{Effects of GM-CSF on the release of SRIF in vitro}

GM-CSF stimulated SRIF release from MBH explants in a doserelated manner (Fig. 6); the dose-response curve was bell-shaped. After a $0.5 \mathrm{hr}$ incubation with $1 \times 10^{-11} \mathrm{M}$ GM-CSF, the release 
Table 2. Sleep parameters before and after $10 \mathrm{pmol}$ of GM-CSF and antibody against GM-CSF

\begin{tabular}{|c|c|c|c|c|c|c|c|c|}
\hline \multirow[b]{3}{*}{ Icv infusion } & \multicolumn{4}{|c|}{ Non-REM sleep } & \multicolumn{4}{|l|}{ REM sleep } \\
\hline & \multicolumn{2}{|c|}{ Episode number } & \multicolumn{2}{|c|}{ Episode duration, min } & \multicolumn{2}{|c|}{ Episode number } & \multicolumn{2}{|c|}{ Episode duration, min } \\
\hline & $\mathrm{L}$ & $\mathrm{D}$ & $\mathrm{L}$ & $\mathrm{D}$ & $\mathrm{L}$ & $\mathrm{D}$ & $\mathrm{L}$ & $\mathrm{D}$ \\
\hline \multicolumn{9}{|c|}{ GM-CSF with 10 pmol $(n=7)$} \\
\hline Day 1 & $162.7 \pm 16.1$ & $97.4 \pm 8.2$ & $3.2 \pm 0.4$ & $2.5 \pm 0.3$ & $64.4 \pm 9.4$ & $20.9 \pm 3.3$ & $1.2 \pm 0.1$ & $1.0 \pm 0.2$ \\
\hline Day 2 & $141.6 \pm 7.4$ & $113.4 \pm 10.5^{*}$ & $3.2 \pm 0.3$ & $2.6 \pm 0.3$ & $56.9 \pm 8.8$ & $41.0 \pm 6.9^{*}$ & $1.4 \pm 0.1$ & $1.2 \pm 0.1$ \\
\hline Day 3 & $170.5 \pm 8.4$ & $111.2 \pm 4.2$ & $2.5 \pm 0.2$ & $2.0 \pm 0.1$ & $72.7 \pm 6.9$ & $33.4 \pm 1.8$ & $1.2 \pm 0.1$ & $1.2 \pm 0.1$ \\
\hline \multicolumn{9}{|c|}{ GM-CSF antibody with $10 \mu \mathrm{g}(n=5)$} \\
\hline Day 1 & $169.2 \pm 7.1$ & $131.0 \pm 6.8$ & $2.6 \pm 0.2$ & $1.9 \pm 0.1$ & $93.4 \pm 8.4$ & $42.9 \pm 7.2$ & $1.1 \pm 0.1$ & $1.4 \pm 0.2$ \\
\hline Day 2 & $165.6 \pm 8.3$ & $108.4 \pm 22.8$ & $2.6 \pm 0.1$ & $2.5 \pm 0.5$ & $81.4 \pm 3.6$ & $35.2 \pm 8.5$ & $1.1 \pm 0.1$ & $1.2 \pm 0.1$ \\
\hline Day 3 & $188.2 \pm 23.0$ & $89.0 \pm 21.2^{*}$ & $2.4 \pm 0.2$ & $1.9 \pm 0.3$ & $71.2 \pm 14.6$ & $22.8 \pm 7.0^{*}$ & $1.9 \pm 0.8$ & $1.2 \pm 0.1$ \\
\hline
\end{tabular}

Values are means \pm SE. L, 12 hr light; D, 12 hr dark.

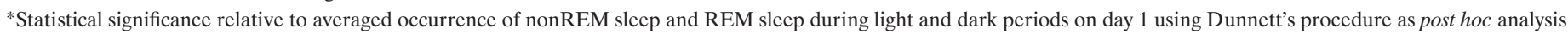
$(p<0.05)$.
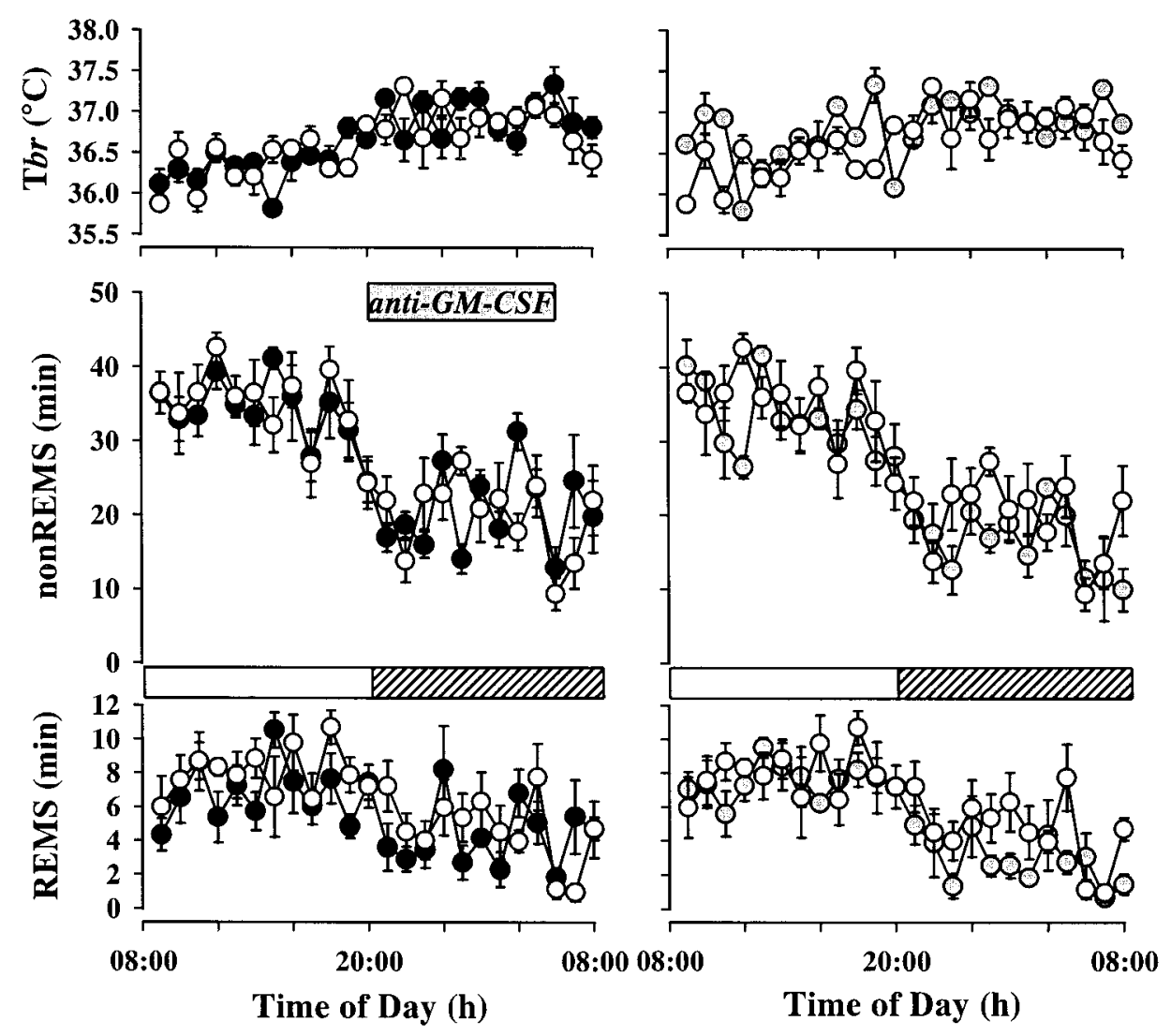

Figure 2. Effects of anti-GM-CSF antibodies $(10 \mu \mathrm{g})$ on sleep and brain temperature $(\mathrm{T} b r)$. Data points indicate $1 \mathrm{hr}$ averages of changes in $\mathrm{T} b r(n=3)$ and time spent in non-REMS and REMS $(n=5)$. Each symbol represents mean \pm SE (white, day 1; black, day 2; gray, day 3). Horizontal gray bar indicates the period when anti-GM-CSF was infused for $10 \mathrm{hr}$ on day 2; otherwise the rats were infused intracerebroventricularly with saline during the entire $3 \mathrm{~d}$ period. Horizontal open bar, Light period; horizontal hatched bar, dark period. ANOVA between days 1 and 2 versus day 3 during the dark period for NREMS, $F_{(2,8)}=$ $5.09 ; p<0.05$, for REMS $F_{(2,8)}=9.24 ; p<$ 0.001 .

of SRIF was doubled compared with that in the control group. However, at any other doses tested, GM-CSF did not alter basal release of SRIF from the $\mathrm{MBH}$.

\section{DISCUSSION}

The results demonstrated that GM-CSF induces significant increases in both non-REM and REM sleep in rats. The effects of GM-CSF on REM sleep were more pronounced than those on non-REM sleep in that REM sleep was promoted for a longer period at a higher magnitude. We are the first to report that a cytokine promotes REM sleep. Most somnogenic cytokines are non-REM sleep promoters and either have no effect on or suppress REM sleep. Thus, the REM sleep-promoting activity of GM-CSF is unique and provides a new perspective of the role of cytokines in sleep regulation. Another important finding is that GM-CSF stimulated non-REM sleep without a concurrent pyrogenic response.
Other immune modifiers such as immune adjuvants induce excess amounts of non-REM sleep in association with fever (Krueger et al., 1987). Although clinical studies have shown febrile responses to either intravenously (Bokemeyer et al., 1993) or subcutaneously (Lopez and Guinan, 1995) administered GM-CSF, the present study found that centrally administered GM-CSF did not cause pyrogenic effects in rats.

Like other cytokines, GM-CSF contributes to the regulation of hormonal release at the level of the pituitary gland (Crispino et al., 1992; Komorowski et al., 1996) and hypothalamus (Bianchi et al., 1997; Kimura et al., 1997). Because hormonal changes often alter sleep patterns (Steiger et al., 1998), GM-CSF might influence or synchronize the endocrine system with its somnogenic activity. To investigate the central effects on endocrine responses in the present study, we tested GM-CSF by intra-Arc injection rather than by intracerebroventricular infusion, because the latter stimulates sev- 


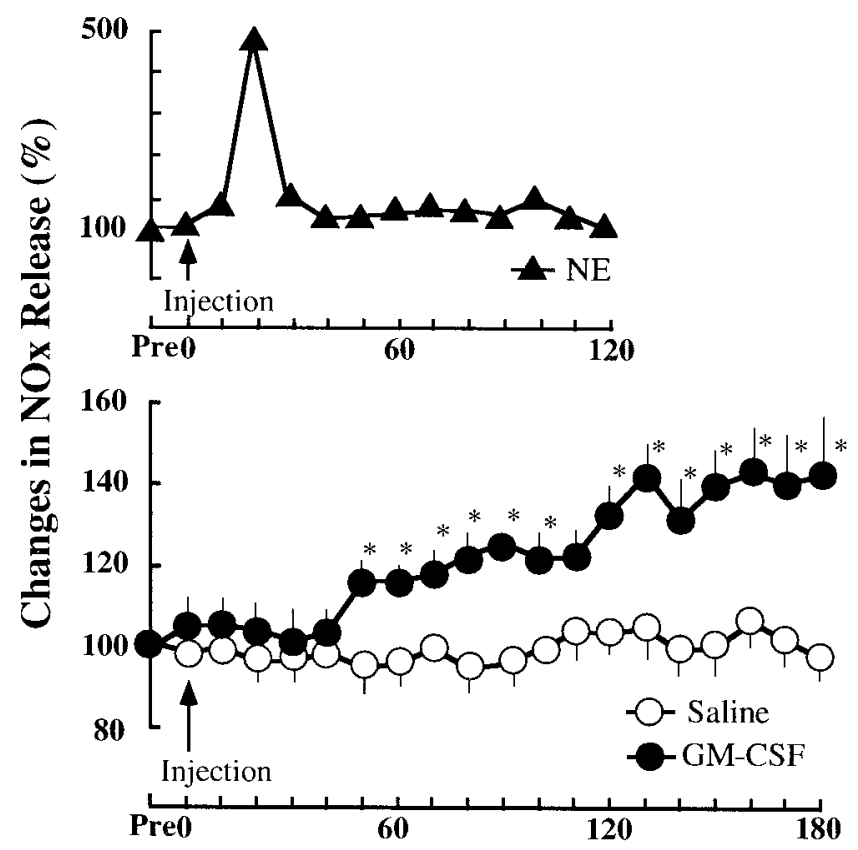

Postinjection Time (min)

Figure 3. Effects of GM-CSF on the release of NOx from the hypothalamus. Top panel, Data points indicate typical changes (\%) in NOx release during and after local administration of norepinephrine (NE; 30 $\mathrm{nmol} / 0.5 \mu \mathrm{l}$ saline; triangles $)$ in the Arc of the hypothalamus, compared with the level before administration (pre). Bottom panel, Data points \pm SE indicate changes $(\%)$ in NOx release during and after local saline administration (open circles) and GM-CSF ( $3 \mathrm{pmol} / 0.5 \mu \mathrm{l}$; solid circles) in the Arc ( $n=6$ each). ANOVA between control and GM-CSFinjected groups $F_{(1,200)}=134.1 ; p<0.001$. ${ }^{*}$ Statistical difference from preinjection baseline; $p<0.05$.

eral groups of neurons around the ventricle, including Arc (Tandon and Sharma, 1985; Rivest et al., 1992), subsequently inducing various CNS responses to the administered substance. The technique is useful when a combination of simultaneous responses from multiple neural circuits is required, but it is not suitable for study- ing a particular response from a specific neuronal group. We previously documented the neuroendocrinological effects of GMCSF on LHRH neurons in the Arc in vitro (Kimura et al., 1997; McCann et al., 1998b). Therefore, here we focused on specific changes in the Arc caused by the direct application of GM-CSF in vivo.

Changes in the Arc NOx content were measured to detect neuroendocrine responses (McCann et al., 1998a). NO is a neurotransmitter gas that stimulates hormonal release (Vallance and Collier, 1994) and most likely participates in the regulation of sleep (Kapás et al., 1994; Burlet et al., 1999). It is immediately converted into NOx after release, which makes measuring NO itself difficult. Therefore, measuring increases or decreases in NOx indicates the dynamics of NO discharge. In the Arc, an NOergic pathway (Bhat et al., 1995) leads NO to activate guanylyl cyclase, and synthesized cGMP induces the secretion of neuropeptides from the hypothalamus (McCann et al., 1998a). The present study showed that a local injection of GM-CSF increased the release of NOx from the Arc indicating the activation of NOergic neurons. In the Arc, the NOergic pathway is involved in the regulation of LHRH (Rettori et al., 1993) and GHRH (Tena-Sempere et al., 1996) release. GHRH and GH are considered sleep-related hormones: GHRH increases both non-REM and REM sleep (Obál et al., 1988; Marshall et al., 1996), and GH predominantly stimulates REM sleep (Drucker-Colin et al., 1975). In the present study, GM-CSF induced REM sleep and also enhanced non-REM sleep. The NOx measurements suggest that GM-CSF stimulates GHRH/GH release and that GHRH and/or GH mediate the sleep promoting activity of GM-CSF.

The results, however, also indicate that $\mathrm{GH}$ secretion is not affected by centrally administered GM-CSF. Therefore, it is unlikely that the somnogenic effects of GM-CSF are mediated by GH. We did not directly measure the release of GHRH in the present study. The fact that $\mathrm{GH}$ secretion was not increased suggests that GM-CSF does not stimulate GHRH release. Plasma levels of GH may also have remained unchanged as a result of the simultaneous secretion of releasing and inhibiting hormones, in which the release of both GHRH and SRIF was stimulated in the hypothalamus. Increased levels of NO in the Arc directly stimulate SRIF synthesis in the periventricular nucleus of the hypothalamus (Aguila, 1994). Our in vitro study demonstrated that GM-CSF induced SRIF release from the MBH. The release of SRIF may be triggered by

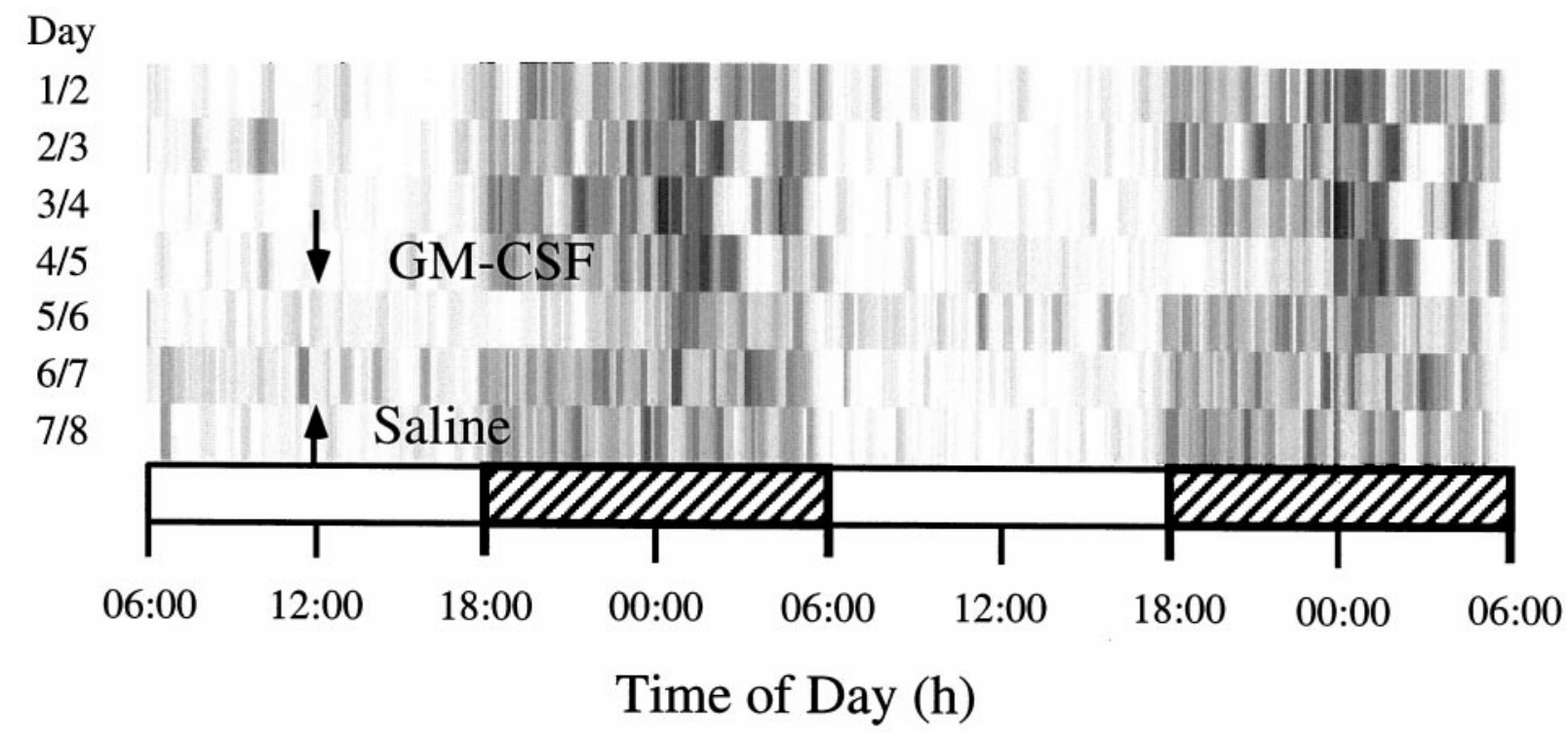

Figure 4. Locomotor activity after the local injection of GM-CSF into the hypothalamus. Locomotor activity was simultaneously monitored while NOx was measured (Fig. 3). Arrows indicate timing of saline or GM-CSF injection. Locomotion intensity is shown as variable densities in vertical columns. Horizontal open bar, Light period; horizontal hatched bar, dark period. 


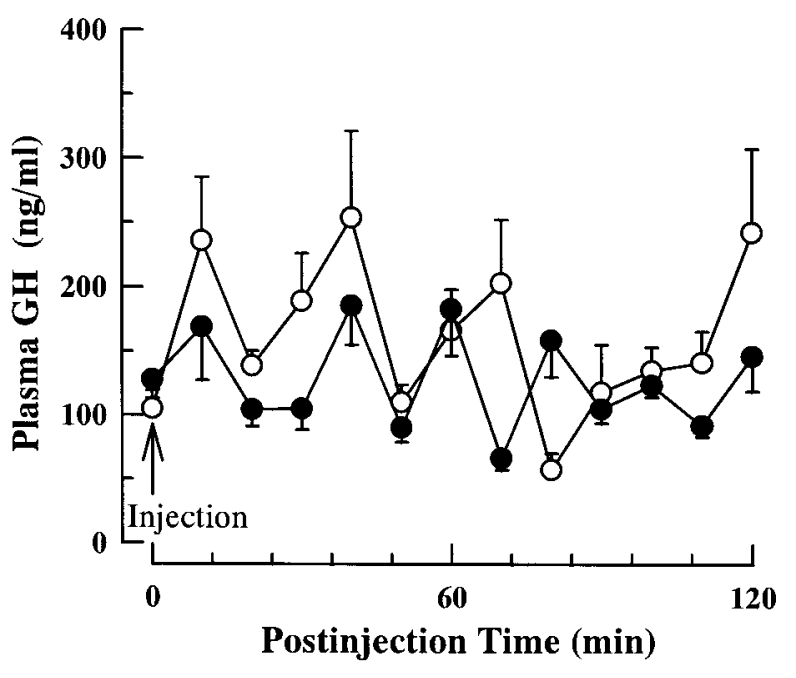

Figure 5. Plasma levels of GH after local GM-CSF injection into the hypothalamus. Data points \pm SEM indicate plasma levels of $\mathrm{GH}$ after local saline and GM-CSF administration (3 pmol/ $/ 0.5 \mu \mathrm{l}$ each) to the Arc of the hypothalamus. ANOVA between control saline and GM-CSF-injected groups $(n=5$ each $), F_{(1,56)}=0.75 ; p>0.05$.

excess NO produced in response to the intracerebroventricular- or intra-Arc administration of GM-CSF.

Indeed SRIF probably mediates the effects of GM-CSF on REM sleep. Intravenously or intracerebroventricularly injected SRIF increases REM sleep without affecting non-REM sleep (Danguir, 1986). Total and REM sleep deprivation increases levels of SRIF mRNA in the rat hypothalamus (Toppila et al., 1996, 1997). Octreotide, an analog of SRIF that induces the long-term inhibition of GH secretion from the pituitary, suppresses non-REM sleep but still enhances REM sleep in rats when systemically administered (Beranek et al., 1997). The intravenous administration of SRIF tends to increase REM density in young adult humans (Steiger et al., 1992). Thus, SRIF may contribute at least in part to the REM sleep-promoting activity of GM-CSF.

Somnogenic cytokines are mostly of inflammatory origin and are responsible for enhanced sleep during infectious diseases. However GM-CSF is not a proinflammatory cytokine produced

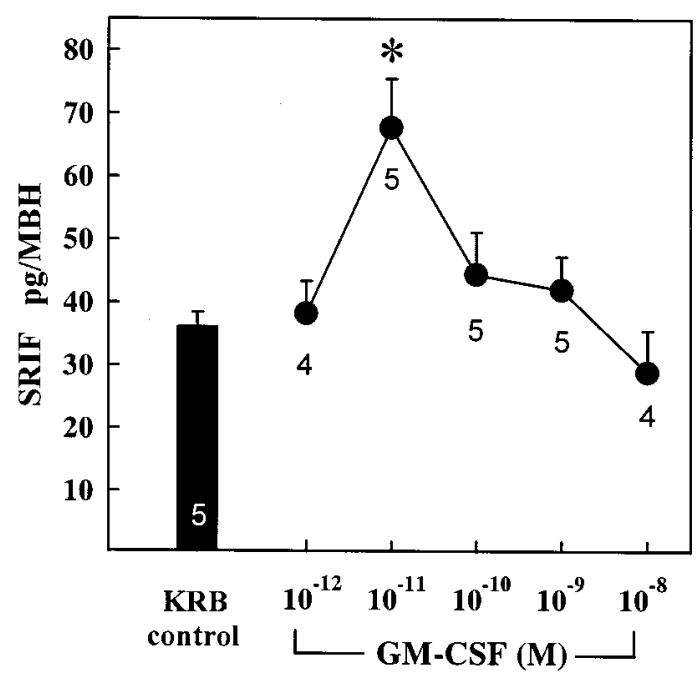

Figure 6. Effects of GM-CSF on SRIF release from the hypothalamus in vitro. Height \pm SE of columns and symbols indicate quantities of SRIF released into the medium incubated without (KRB only) or with GM-CSF. Numbers represent number of explants of the MBH in each group. ANOVA among all groups, $F_{(2,23)}=4.04 ; p<0.01$. * Statistical difference from KRB control; $p<0.05$. immediately after the onset of infection or inflammation. Rather, it is induced after the cascade activation of proinflammatory cytokines, including somnogenic IL-1 (Henricson et al., 1991) and TNF- $\alpha$ (Duru et al., 1995). Only GM-CSF promotes REM sleep, whereas other cytokines that stimulate GM-CSF release do not. The lack of REM sleep-promoting effects of IL-1 and TNF- $\alpha$ seems to be a masking effect. The effects of IL-1 or TNF- $\alpha$ on non-REM sleep and temperature are very powerful (Dinarello, 1999) and may mask the effects of subsequently released GM-CSF on REM sleep. The central effects of GM$\mathrm{CSF}$ are milder than most cytokines used in clinical practice, such as IL-1, TNF- $\alpha$, and interferon. One of the problems with the therapeutic use of these cytokines is that of neurological side effects similar to "flu-like" symptoms, including fever, nausea, and sleepiness (Eskander et al., 1997). Because CSFs are less toxic and do not heavily disturb the natural composition of sleep-wake patterns, the benefits of treatment with these factors could be clinically applied.

Our results support the notion that GM-CSF functions not only as a hemopoietic growth factor but that it also plays a role in the regulation of endocrine function, behavior, and vigilance like the other two CSFs. Whereas centrally administered M-CSF affects REM sleep in rats (Kimura et al., 1998b), systemically injected G-CSF suppresses sleep intensity in humans (Schuld et al., 1999). Although the effects of exogenous CSFs on sleep have been demonstrated, the significance of endogenous CSFs in sleep regulation needs to be further elucidated. During pregnancy, CSFs are crucial to support the growth and implantation of the fertilized egg. In early pregnancy, sleep and sleepiness increase in humans (American Sleep Disorders Association, 1990; Mauri, 1990; Driver and Shapiro, 1992) and rats (Kimura et al., 1996b, 1998a); therefore, the role of GM-CSF in pregnancy-altered sleep should be an appropriate topic of study. Moreover CSFs in the CNS and PNS promote neurite outgrowth and neuronal differentiation while the fetus undergoes neural development (Mehler and Kessler, 1997, 1998). During later development, CSFs disappear from the CNS, but their receptors remain on the surface of the glial cells in the adult brain (Sei et al., 1995). The receptors specific to GM-CSF located on the microglia consist of two subunits sharing a common $\beta c$ chain with IL-3 and IL-5 (Weiss et al., 1993). The activation of Janus and src-family cytoplasmic tyrosine kinases appears to be a critical event in GM-CSF signaling (Quelle et al., 1994). As described above, hemopoietic cytokines, like interleukins, can function as neuromodulators or as neurotropins in the brain under certain physiological and pathophysiological conditions (Kamegai et al., 1990; Bhat et al., 1995). Although GM-CSF is usually produced peripherally, it may provide a signal to the CNS by crossing the blood-brain barrier (McLay et al., 1997) or act on neurons located near the circumventricular organs (Helson et al., 1993). Although how GM-CSF sends neurochemical signals to neurons or a specific nucleus of the brain remains unknown, the results of the present study are consistent with the notion that hematopoietins can be neural mediators.

Few investigators have examined the role of CSFs in the brain. The present study adds the novel findings that GM-CSF promotes non-REM and REM sleep in the rat.

\section{REFERENCES}

Aguila MC (1994) Growth hormone-releasing factor increases somatostatin release and mRNA levels in the rat periventricular nucleus via nitric oxide by activation of guanylate cyclase. Proc Natl Acad Sci USA 91:782-786.

Aguila MC, McCann SM (1985) Stimulation of somatostatin release in vitro by synthetic human growth hormone releasing factor by a nondopaminergic mechanism. Endocrinology 117:762-765.

Aguila MC, Pickle R, Yu WH, McCann SM (1991) Roles of somatostatin (SRIF) and growth hormone releasing factor (GRF) in ether stress inhibition of growth hormone $(\mathrm{GH})$ release. Neuroendocrinology 54:515-520.

Agullo L, Garcia A (1991) Norepinephrine increases cyclic GMP in 
astrocytes by a mechanism dependent on nitric oxide synthesis. Eur J Pharmacol 206:343-346.

Aloisi F, Carè A, Borsellino G, Gallo P, Rosa S, Bassani A, Cabibbo A Testa U, Levi G, Peschile C (1992) Production of hemolymphopoietic cytokines (IL-6, IL-8, colony-stimulating factors) by normal human astrocytes in response to IL $-1 \beta$ and tumor necrosis factor- $\alpha$. J Immunol 149:2358-2366.

American Sleep Disorders Association (1990) The international classification of sleep disorders: diagnostic and cording manual, Ed 1, pp 297-300. Rochester, NY: American Sleep Disorders Association.

Arimura A, Sato H, Coy DH, Schally AV (1975) Radioimmunoassay of GH-release inhibiting hormones. Proc Soc Exp Biol Med 148:784-789

Baldwin GC, Benveniste EN, Chung GY, Gasson JC, Golde DW (1993) Identification and characterization of a high-affinity granulocytemacrophage colony-stimulating factor receptor on primary rat oligodendrocytes. Blood 82:3279-3282.

Beranek L, Obál Jr F, Taishi P, Bodosi B, Laczi F, Krueger JM (1997) Changes in rat sleep after single and repeated injections of the long-acting somatostatin analog octreotide. Am J Physiol 273:R1484-R1491.

Bhat GK, Mahesh VB, Lamar CA, Ping L, Aguan K, Brann DW (1995) Histochemical localization of nitric oxide neurons in the hypothalamus: association with gonadotropin-releasing hormone neurons and co-localization with $N$-methyl-D-aspartate receptors. Neuroendocrinology 62:187-197.

Bianchi M, Clavenna A, Bondiolotti GP, Ferrario P, Panerai AE (1997) GM-CSF affects hypothalamic transmitter levels in mice: involvement of interleukin-1. NeuroReport 8:3587-3590.

Bokemeyer C, Schmoll H-J, Harstrick A (1993) Side-effects of GMCSF treatment in advanced testicular cancer. Eur J Cancer 29A:924.

Burgess AW, Metcalf D (1980) The nature and action of granulocyte macrophage colony-stimulating factor. Blood 56:947-958.

Burlet S, Leger L, Cespuglio R (1999) Nitric oxide and sleep in the rat: a puzzling relationship. Neuroscience 92:627-639.

Crispino S, Lissoni P, Ardizzoia A, Barni S, Rovelli F, Tancini G (1992) Effects of granulocyte-macrophage colony stimulating factor on cortisol, growth hormone, prolactin and melatonin in cancer patients. J Biol Regul Homeost Agents 6:142-144.

Danguir J (1986) Intracerebroventricular infusion of somatostatin selectively increases paradoxical sleep in rats. Brain Res 367:26-30.

Dinarello CA (1999) Cytokines as endogenous pyrogens. J Inf Dis [Suppl 2] 179:S294-S304.

Driver HS, Shapiro CM (1992) A longitudinal study of sleep stages in young women during pregnancy and postpartum. Sleep 15:449-453.

Drucker-Colin RR, Spanis CW, Hunyadi J, Sassin JF, McGaugh JL (1975) Growth hormone effects on sleep and wakefulness in the rat. Neuroendocrinology 18:1-8.

Duru F, Ertem U, Tacyildiz N, Kirazli S (1995) Production of granulocyte colony stimulating factor, granulocyte macrophage colony stimulating factor, and tumor necrosis factor alpha during remission and infections in patients with acute leukemia. J Med 26:241-252.

Eskander ED, Harvey HA, Givant E, Lipton A (1997) Phase I study combining tumor necrosis factor with interferon-alpha and interleukin-2. Am J Clin Oncol 20:511-514.

Fischer HG, Nitzgen B, Germann T, Degitz K, Däubener W, Hadding U (1993) Differentiation driven by granulocyte-macrophage colonystimulating factor endows microglia with interferon- $\gamma$-independent antigen presentation function. J Neuroimmunol 42:87-96.

Giulian D, Li J, Li X, George J, Rutecki PA (1994) The impact of microglia-derived cytokines upon gliosis in the CNS. Dev Neurosci $16: 128-136$.

Helson L, Braverman S, Rifkinson S, Helson C (1993) Cerebral ventricular fluid distribution of subcutaneous granulocyte-macrophage colony stimulating factor. Anticancer Res 13:1851-1856.

Henricson BE, Neta R, Vogel SN (1991) An interleukin-1 receptor antagonist blocks lipopolysaccharide-induced colony-stimulating factor production and early endotoxin tolerance. Inf Immun 52:1188-1191.

Inoué S, Honda K, Komoda Y (1985) Sleep-promoting substances. In: Sleep: neurotransmitters and neuromodulators (Wauquier A, Gaillard JM, Monti J, Radulovacki M, eds), pp 305-318. New York: Raven.

Kamegai M, Konishi Y, Tabira T (1990) Trophic effect of granulocytemacrophage colony-stimulating factor on central cholinergic neurons in vitro. Brain Res 532:323-325.

Kapás L, Shibata M, Kimura M, Krueger JM (1994) Inhibition of nitric oxide synthesis suppresses sleep in rabbits. Am J Physiol 266:R151-R157.

Karanth S, Lyson K, McCann SM (1993) Role of nitric oxide in interleukin 2-induced corticotropin-releasing factor release from incubated hypothalami. Proc Natl Acad Sci USA 90:3383-3387.

Kimura M, Yu WH, McCann SM (1996a) Centrally administrated granulocyte-macrophage colony stimulating factor stimulates $\mathrm{LH}$ but suppresses FSH release in ovariectomized female rats. 10th Intl Congress of Endocrinol Abstr 1:539.

Kimura M, Zhang S-Q, Inoué S (1996b) Pregnancy-associated sleep changes in the rat. Am J Physiol 271:R1063-R1069.
Kimura M, Yu WH, Rettori V, McCann SM (1997) Granulocytemacrophage colony stimulating factor (GM-CSF) suppresses LHRH release by inhibition of nitric oxide synthase and stimulation of gammaaminobutyric acid release. Neuroimmunomodulation 4:237-243.

Kimura M, Zhang S-Q, Inoué S (1998a) An animal model for pregnancy-associated sleep disorder. Psychiatry Clin Neurosci 52:209-211.

Kimura M, Kodama T, Aguila MC, Zhang S-Q, Inoué S (1998b) REM sleep-inducing cytokines: colony-stimulating factors. J Sleep Res [Suppl 2] 7:135.

Komorowski J, Zylinska K, Mucha S, Robak T, Wrzesien-Kus A, Stepien H (1996) Effect of granulocyte-macrophage colony stimulating factor and granulocyte colony stimulating factor on prolactin and adrenocorticotropic hormone secretion in rats: dose- and timeresponse in vivo studies. Cytobios 86:147-153.

Krueger JM, Majde JA (1994) Microbial products and cytokines in sleep and fever regulation. Crit Rev Immunol 14:355-379.

Krueger JM, Davenne D, Walter J, Shoham S, Kubillus SL, Rosenthal RS, Martin SA, Biemann K (1987) Bacterial peptidoglycan as modulators of sleep. II Effects of muramyl peptides on the structure of rabbit sleep. Brain Res 403:258-266.

Kuhns DB, Alvord WG, Gallin JI (1995) Increased circulating cytokines, cytokine antagonists, and E-selectin after intravenous administration of endotoxin in humans. J Inf Dis 171:145-152.

Lieschke GJ, Maher D, O’Connor M, Green M, Sheridan W, Rallings M, Bonnem E, Burgess AW, McGrath K, Fox RM, Morstyn G (1990) Phase I study of intravenously administered bacterially synthesized granulocyte-macrophage colony-stimulating factor and comparison with subcutaneous administration. Cancer Res 50:606-614.

Lopez KD, Guinan EC (1995) GM-CSF clinical trials: pediatric aplastic anemia and Fanconi's anemia. Pediatr Nurs 21:345-349.

Malipiero UV, Frei K, Fontana A (1990) Production of hemopoietic colony-stimulating factors by astrocytes. J Immunol 144:3816-3821.

Marshall L, Molle M, Boschen G, Steiger A, Fehm HL, Born J (1996) Greater efficacy of episodic than continuous growth hormonereleasing hormone $(\mathrm{GHRH})$ administration in promoting slow-wave sleep (SWS). J Clin Endocrinol Metab 81:1009-1013.

Mauri M (1990) Sleep and the reproductive cycle: a review. Health Care Women Int 11:409-421.

McCann SM, Karanth S, Dees WL, Lyson K, Gimeno M, Rettori V (1994) Induction by cytokines of the pattern of pituitary hormone secretion in infection. Neuroimmunomodulation 1:2-13.

McCann SM, Kimura M, Karanth S, Yu WH, Rettori V (1998a) Role of nitric oxide in the neuroendocrine responses to cytokines. Ann NY Acad Sci 840:174-184.

McCann SM, Kimura M, Walczewska A, Karanth S, Rettori V, Yu WH (1998b) Hypothalamic control of gonadotropin secretion by LHRH, FSH-RF, NO, cytokines, and leptin. Domest Anim Endocrinol $15: 333-344$

McLay RN, Kimura M, Banks WA, Kastin AJ (1997) Granulocytemacrophage colony stimulating factor crosses the blood-brain and blood-spinal cord barriers. Brain 120:2083-2091.

Mehler MF, Kessler JA (1997) Hematolymphopoietic and inflammatory cytokines in neural development. Trends Neurosci 20:357-365.

Mehler MF, Kessler JA (1998) Cytokines in brain development and function. Adv Protein Chem 52:223-251.

Obál Jr F, Alföldi P, Cady AB, Johannsen L, Sáry Gy, Krueger JM (1988) Growth hormone-releasing factor enhances sleep in rats and rabbits. Am J Physiol 255:R310-R316.

Paxinos G, Watson C (1997) The rat brain in stereotaxic coordinates, Ed 3. San Diego: Academic.

Quelle FW, Sato N, Witthuhn BA, Inhoru RC, Eder M, Miyajima A, Griffin JD, Ihle JN (1994) JAK2 associates with the beta (c) chain of the receptor for granulocyte-macrophage colony-stimulating factor, and its activation requires the membrane-proximal region. Mol Cell Biol 14:4335-4341.

Rettori V, Belova N, Dees WL, Nyberg CL, Gimeno M, McCann SM (1993) Role of nitric oxide in the control of luteinizing hormonereleasing hormone release in vivo and in vitro. Proc Natl Acad Sci USA 90:10130-10134.

Rivest S, Torres G, Rivier C (1992) Differential effects of central and peripheral injection of interleukin-1 beta on brain c-fos expression and neuroendocrine functions. Brain Res 587:13-23.

Robertson SA, Seamark RF (1992) Granulocyte-macrophage colony stimulating factor (GM-CSF): one of a family of epithelial cellderived cytokines in the preimplantation uterus. Reprod Fertil Dev 4:435-448.

Rothwell NJ, Hopkins SJ (1995) Cytokines and the nervous system II: actions and mechanisms of action. Trends Neurosci 18:130-136.

Sawada M, Itoh Y, Suzumura A, Marunouchi T (1993) Expression of cytokine receptors in cultured neuronal and glial cells. Neurosci Lett 160:131-134.

Schuld A, Mullington J, Hermann D, Hinze-Selch D, Fenzel T, Holsboer F, Pollmächer T (1999) Effects of granulocyte colony-stimulating factor on night sleep in humans. Am J Physiol 276:R1149-R1155.

Sei Y, Vitkovic L, Yokoyama MM (1995) Cytokines in the central 
nervous system: regulatory roles in neuronal function. cell death and repair. Neuroimmunomodulation 2:121-133.

Steiger A, Guldner J, Hemmeter U, Rothe B, Wiedemann K, Holsboer F (1992) Effects of growth hormone-releasing hormone and somatostatin on sleep EEG and nocturnal hormone secretion in male controls. Neuroendocrinology 56:566-573.

Steiger A, Antonijevic IA, Bohlhalter S, Frieboes RM, Friess E, Murck H (1998) Effects of hormones on sleep. Horm Res 49:125-130.

Tandon OP, Sharma KN (1985) Effect of third ventricular injection of beta-endorphin on the electrophysiological responses of some regions of endocrine hypothalamus. Indian J Physiol Pharmacol 29:75-82.

Tena-Sempere M, Pinilla L, Gonzalez D, Aguilar E (1996) Involvement of endogenous nitric oxide in the control of pituitary responsiveness to different elicitors of growth hormone release in prepubertal rats. Neuroendocrinology 64:146-152.

Toppila J, Asikainen M, Alanko L, Turek FW, Stenberg D, Porkka-
Heiskanen T (1996) The effect of REM sleep deprivation on somatostatin and growth hormone-releasing hormone gene expression in the rat hypothalamus. J Sleep Res 5:115-122.

Toppila J, Alanko L, Asikainen M, Tobler I, Stenberg D, PorkkaHeiskanen T (1997) Sleep deprivation increases somatostatin and growth hormone-releasing hormone messenger RNA in the rat hypothalamus. J Sleep Res 6:171-178.

Vallance P, Collier J (1994) Biology and clinical relevance of nitric oxide. Br Med J 309:453-457.

Weiss M, Yokoyama C, Shikama Y, Naugle C, Druker B, Sieff CA (1993) Human granulocyte-macrophage colony-stimulating factor receptor signal transduction requires the proximal cytoplasmic domains of the $\alpha$ and $\beta$ subunits. Blood 82:3298-3306.

Yamada K, Senzaki K, Komori Y, Nikai T, Sugihara H, Nabeshima T (1997) Changes in extracellular nitrite and nitrate levels after inhibition of glial metabolism with fluorocitrate. Brain Res 762:72-78. 